\title{
New Method for the Detection of $T$ Wave Alternans in Basis of Walsh Functions
}

\author{
OV Melnik
}

\author{
Ryazan State Radioengineering University, Russia
}

\begin{abstract}
For the detection and analysis of T-wave alternans (TWA) is offered use the integral method based on the Walsh functions spectral amplitudes calculation.

Spectral transformation in basis of Walsh functions was produced in time-domain window with $132 \mathrm{~ms}$ duration, located symmetrically to energy center of the $S T / T$-wave in each heartbeat. Values of spectral amplitudes are possible to consider as qualitative and quantitative morphology parameters. The comparison of morphology parameters of T-wave of consequent heartbeats allows to reveal microalternans.

Algorithms and software for detection the energy center of T-wave in each heartbeat, calculations morphology parameters and identification of T-wave alternans were designed.

The offered method is characterized by high noise proof, does not depend on heart rate and can reveal not only two but more distinct forms of T-waves.
\end{abstract}

\section{Introduction}

Changes of amplitude-temporary parameters of electrocardiosignal (ECS) at area corresponding to cardiocycle repolarization phase are an important diagnostic sign of myocardial diseases. Beat-to-beat $\mathrm{T}$ wave microalternans are significant mark of the electric instability of the myocardium, which can lead to ventricular arrythmia and sudden cardiac death.

Methods of direct measurement of amplitudetemporary parameters of T-wave do not provide of acceptable accuracy of measurements. It is joined to real ECS acting an additive handicaps, on amplitude comparable with a useful signal. The presence of handicaps is not traced and can result in failures in work of diagnostic algorithms and, so, to statement of the erroneous diagnosis by automatic system.

For T-wave microalternans estimation the class of the FFT spectral methods is most wide-spread. But given methods are limited by requirement to low noise ECS registration (signal-to-noise ratio $>3$ ) and require accumulations of the big amount of heartbeats with
TWA. These methods do not allow to analyse short-time ECS records and reveal the short episodes of the TWA appearance.

For decision of the problem of increasing of validity of T-wave alternans detection in each consequent heart-beat is offered to use the approach, based on calculation of integrated parameters [1], such as spectral coefficients of a signal of T-wave in Walsh functions basis.

\section{Methods}

From the medical point of view of the most important clinical parameters at research such ECS element as a Twave are a degree and sign of displacement from iso-line and form, which in a general view can be described as a superposition of slopping and nonlinear components. Therefore at a choice of basis of spectral representation it is necessary to be guided by presence of clear interrelation between the specified amplitude-temporary attributes and spectral structure of a signal. Using the theory of detection of signals there was stated that to the best advantage supplied requirements satisfy the basis of Walsh functions.

It was declared that the zero $\mathrm{W}_{0}$, the first $\mathrm{W}_{1}$ and the third $\mathrm{W}_{3}$ spectral amplitudes in Walsh basis ranked on Paly characterize, accordingly, degree and sign of offset, direction of slopping and presence of proturberance or concavities [2]. Values of given spectral amplitudes possible to consider as morphology parameters - offsets (PO), slopping (PS) and proturberances/concavities $(\mathrm{PP} / \mathrm{C})$.

Existence of constant coefficients of intercoupling between spectral amplitudes values and morphology parameters allows not only qualitative, but also quantitative to value it. The factors of issue each of morphology parameters $\mathrm{K}$ on corresponding to spectral amplitudes are presented in Table 1.

Table 1. Factors of issue.

\begin{tabular}{|c|c|c|c|}
\hline & $\mathrm{PO} / \mathrm{K}_{0}$ & $\mathrm{PS} / \mathrm{K}_{1}$ & $\mathrm{PP} / \mathrm{C} / \mathrm{K}_{2}$ \\
\hline Walsh & $\mathrm{W}_{0} / 1$ & $\mathrm{~W}_{1} /-0,5$ & $\mathrm{~W}_{3} / 0,375$ \\
\hline
\end{tabular}

Thereby, set of three spectral amplitudes $\mathrm{W}_{0}, \mathrm{~W}_{1}$ and $\mathrm{W}_{3}$ allow to characterize the main parameters of the form 
of the ECS element, including T-wave. At fig. 1 different variants of the T-wave form and quantitative and qualitative characterizing its set of the spectral coefficients are presented. If the T-wave is symmetrical from point of the medium of the interval of $\mathrm{T}$-wave existence TC between beginning TB and completion TE of T-wave (fig. 1, a, b), when magnitude and sign of $\mathrm{W}_{0}$ reflect the amplitude and position of $\mathrm{T}$-wave comparatively to iso-line; magnitude and sign of $\mathrm{W}_{3}$ rate and type of proturberances or concavities. The spectral amplitude $\mathrm{W}_{1}$ in this case is a zero. But if $\mathrm{T}$ wave maximum is displaced from the centre of the $\mathrm{T}$ wave interval (fig. 1, c, d), then sign and magnitude of the $\mathrm{W}_{1}$ spectral amplitude allows to measure the direction and value of offset. Magnitude and sign of $\mathrm{W}_{3}$ amplitude, either as in previous event, are characterized rate and type of proturberances or concavities.
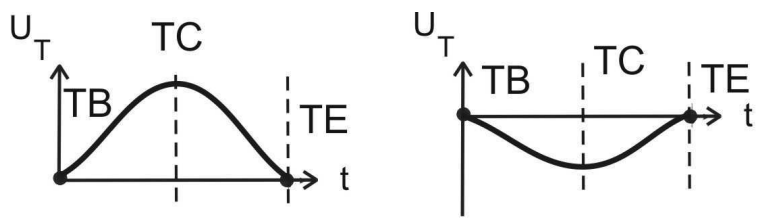

a

b

$\mathrm{W}_{\mathrm{k}}$

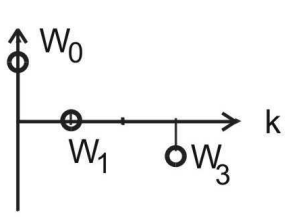

W

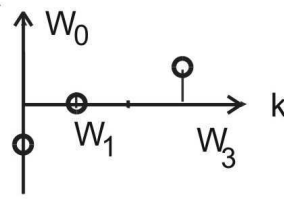

$\mathrm{U}$

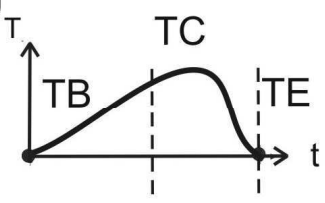

$\mathrm{U}_{\mathrm{T}}$

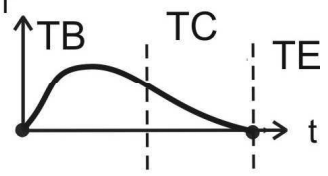

C

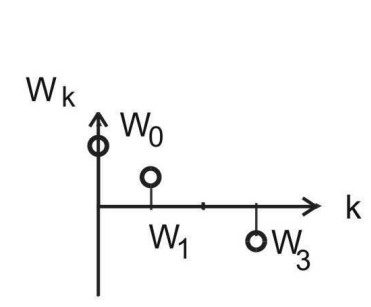

d

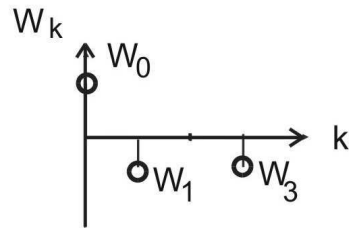

Figure 1. Variants of the T-wave forms and its spectral amplitudes in Walsh basis.

Values of the spectral amplitudes in each heartbeat characterize the particularities of the each T-wave form. The got morphology parameters also can be used for inverse transform that is to say for recovering main sign of time-domain form of the T-wave cleaned from highfrequency noise handicaps.

For the detection of TWA such algorithm was offered.

1. ECS filtering for iso-line drift elimination.

2. Allocation of ST segment borders. There was offered method of "QRS-strobing" of ST segment signal [3]. Method of QRS-strobing is founded on that that at summation of first derived energy in driven time-domain window, duration $W W$ is equval with duration QRScomplex $T_{Q R S}$, the denominated maximum of function is reached at moment of completion QRS-complex then in point of ST segment begin. At excess of duration $W W$ to $T_{Q R S}$ point of maximum convert to "ledge" of constant level by duration, equal duration $W W$ subtract QRScomplex duration. At choice of duration of time-domain window equal $T_{S T}+T_{Q R S}$ will is formed strobe pulse, coinsiding with ST-segment time interval. Given method allows to abandon of searching for fidusial point within QRS-complex (R-peak). For ECS morphologies variability account are offered to use the dynamic turning of duration of time-domain window, applying of additional control functions and dynamic adaptive thresholds of comparison. This method ensures steady work in a real time mode in the conditions of amplitudetemporary parameters variability of ECS waves and complexes.

3. Energy center of the ST/T-wave detection in each heartbeat. After finding the point, considered as the end of ST segment, it is produced searching for of the local maximum of energy at $140 \mathrm{~ms}$ interval. Searching for the most magnitude of averaged energy of signal in driven time-domain window, duration equal minimum achievable T-wave duration is formed. Founded maximum is considered as energy centre of T-wave and not always complies with maximum of $\mathrm{T}$-wave amplitude. On possibility of the finding of the energy centre do not affect polarity and form of T-wave. On accuracy of the localizations do not affect high-frequency noise handicaps, as in the case of searching for of the maximum of T-wave amplitude.

4. Walsh spectral amplitudes calculation. In each heartbeat calculation of $\mathrm{W}_{0}, \mathrm{~W}_{1}$ and $\mathrm{W}_{3}$ coefficients is produced in time-domain window with $132 \mathrm{~ms}$ duration, located symmetrically to energy center of the ST/T-wave. In general event number of discrete samples $m$, need for describing of three specified discrete Walsh functions must be multiple of 4 . The minimum number of discrete samples, which possible to present T-wave after ECS sampling is defined by attitude to minimum $\mathrm{T}$-wave duration $\mathrm{T}_{\mathrm{Tmin}}$ to period of the sampling $\Delta t$. Since number 
$N$ of discrete samples can be only integer number and, in accordance with foregoing, multiple of 4 , that it must be defined from condition

$$
N=m \cdot \operatorname{int}\left(\frac{\operatorname{int}\left(\frac{T_{T \min }}{\Delta t}\right)}{m}\right)
$$

where $\mathrm{T}_{\mathrm{Tmin}}$ is minimum T-wave duration, $\mathrm{T}_{\mathrm{Tmin}}=132 \mathrm{~ms}$; $\operatorname{int}(a / b)$ means taking an integer from division a to $b$.

For sample rate 500 sample per second $N=64 . N$ values of ECS samples $u_{\mathrm{i}}$ used for $\mathrm{W}_{0}, \mathrm{~W}_{1}$ and $\mathrm{W}_{3}$ amplitudes calculation:

$$
\begin{aligned}
& W 0_{k}=\frac{1}{N} \sum_{i=k-N}^{k} u_{i}, \\
& W 1_{k}=\frac{1}{N}\left[-\sum_{i=k-N}^{k-\frac{N}{2}} u_{i}+\sum_{i=k-\frac{N}{2}+1}^{k} u_{i}\right. \text {, } \\
& W 3_{k}=\frac{1}{N}\left[\sum_{i=k-N}^{k-\frac{3 N}{4}} u_{i}-\sum_{i=k-\frac{3 N}{4}+1}^{k-\frac{N}{4}} u_{i}+\sum_{i=k-\frac{N}{4}+1}^{k} u_{i}\right]
\end{aligned}
$$

It was declared [4] that narrowing of the time-domain interval, on which are spectral amplitudes calculated, by comparison with borders of the ECS element does not provide the influences upon qualitative character of the spectrum, unlike event, when duration of time-domain window exceeds duration of the element.
Thereby, choice of the size of time-domain window close to minimum duration $\mathrm{T}_{\mathrm{Tmin}}$ is preferred and more universal in the event of different $\mathrm{T}$-wave duration of various patients' records.

Got values $\mathrm{W}_{0}, \mathrm{~W}_{1}, \mathrm{~W}_{3}$ are saved for the further processing.

4. Reconstruction of time-domain form of each Twave. Reconstruction of amplitudes of 4 samples of Twave based on morphology parameters $\mathrm{W}_{0}, \mathrm{~W}_{1}, \mathrm{~W}_{3}$ is produced. Got values are characterizing each T-wave form, cleaned from high-frequency noise and unimportant distortions.

5. Alternans calculation in each heartbeat. For each two consequent heartbeats maximum difference between got magnitudes of the restored signal is found. These values are considered as an amount of alternans.

6. Detecting of the peak magnitude of alternans. For whole ECS record maximum magnitude of alternans between two consequent heartbeats is fixed.

7. Estimation of the average beat-to-beat magnitude of alternans. The calculation of average magnitude of alternans in all even and uneven numbers of heartbeat is realized. Difference between given two values is the average alternans in full ECS record.

\section{Results}

A new approach to TWA detection was declared. The program realizing the set of the offered methods and allowing to make an estimation of a T-wave alternans has been developed use Borland Delphi 7.0. Window of the program presented at fig. 2 .

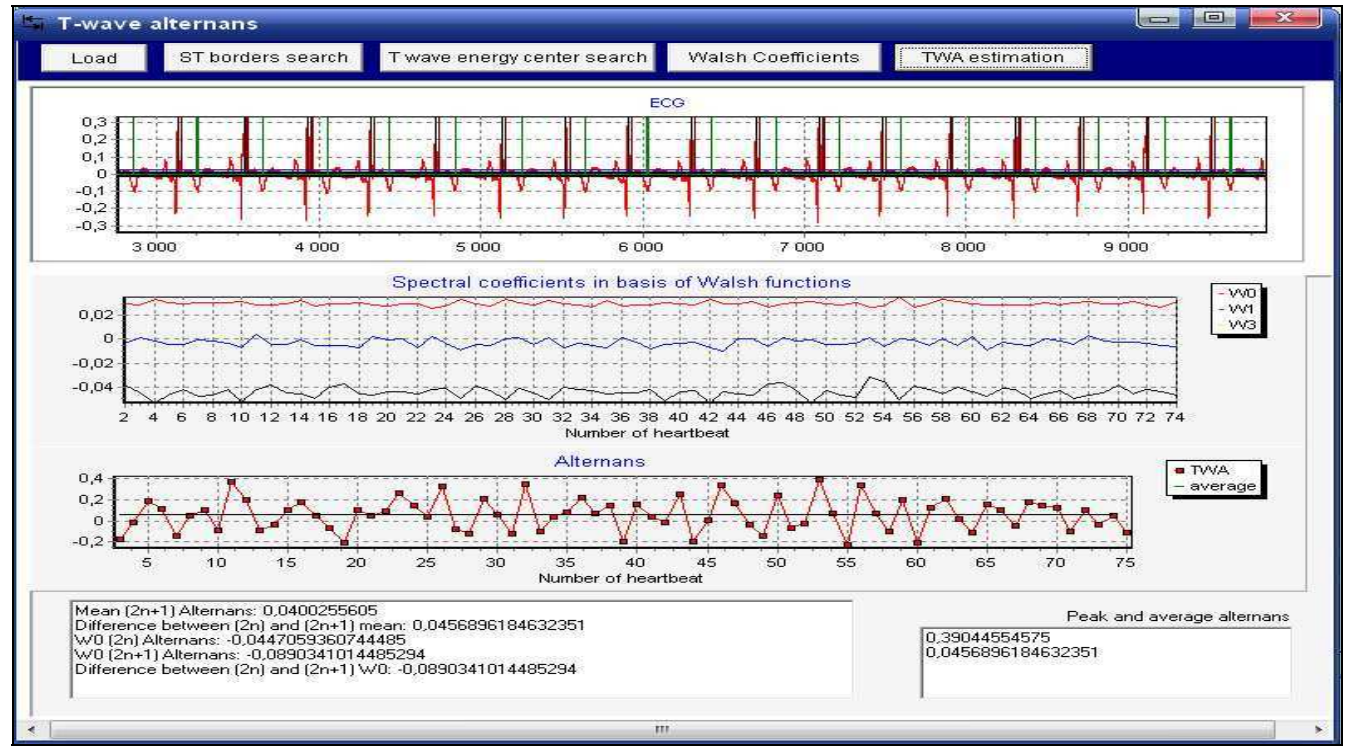

Figure 2. Program for TWA detection. Charts of ECS, Walsh spectral amplitudes and T-wave alternans (microvolt). 
Functioning of the program was tested at 100 ECS records from T-Wave Alternans Challenge Database.

\section{Discussion and conclusions}

The method of revealing of $\mathrm{T}$-wave alternans use spectral structure of T-wave in basis of Walsh functions has been developed and investigated. In the comparison with direct measurement wave amplitude, it provides a 68 times larger stability to the influence of the noise handicapes on ECS, that raises sensitivity to the low-level changes of the morphology of a T-wave and the shortterm deviations as well. The method allows graphically tracing changes of the form of T-wave in each heartbeat and calculating average beat-to-beat magnitude of alternans. It suits for analysis of the record to any even very short duration and does not depend on type of the ECS lead.

The study called on T-Wave Database was shown that is not always possible to distinguish full automatically alternans and ECS distortion, conditioned noise. The ways of increasing of reliability of given method are the more qualitative preliminary filtering of ECS and the development of the algorithm of interpretation of sequences and trends of alternans magnitudes.

\section{References}

[1] Melnik O.V. Development of the automatic system for complex ischemia diagnostics and the risk of sudden cardiac death revealing. BIOMEDSYSTEMS-2007:242-5.

[2] Melnik G.I, Melnik O.V., Philatov A.D. The integrated approach to an estimation of conditional-determined signals parameters. The bulletin of Moskow State Open University (New technologies):2003:57-3.

[3] RF Patent 2261653 (2005) A 61 B5/0402, B5/0452 O.V. Melnik, A.A. Miheev Method of ST segment time-domain interval detection and device for its realization.

[4] Melnik O.V., Miheev A.A. Integral way to estimation of ST segment of electrocardiosignal parameters, Biomedical technology and radioengineering: 2003: 5: 8-4.

O.V. Melnik

Biomedical and Semiconductor Electronics Department Ryazan State Radioengineering University

Gagarina, 59/1

Ryazan, Russia

390005

omela111@yandex.ru 